\title{
LA MIGRACION INTERNACIONAL EN ARGENTINA HACIA 2010
}

Laura Calvelo*

\begin{abstract}
El artículo presenta los principales lineamientos de la migración internacional en Argentina hacia el año 2010, con detenimiento en el período 2000-2010. En el caso de la inmigración no nativa se basa en información de los censos nacionales de población 2001 y 2010. En el caso de la migración internacional de la población nativa de Argentina el análisis se sustenta en información de censos extranjeros hasta la ronda 2000 y en otras fuentes de datos de los principales países de destino hasta 2010 (Estados Unidos y España).
\end{abstract}

Palabras clave: Migración Internacional en Argentina; Inmigración no nativa en Argentina; Emigracion nativa en Argentina; Medición de la Migración Internacional en Argentina.

\section{La migración internacional en Argentina en la última década}

En este artículo se repasan los principales lineamientos de la migración internacional 2001-2010 en Argentina, un país donde el movimiento internacional de población ha sido en el pasado y sigue siendo en el presente un factor decisivo en la formación de los mercados de trabajo y en la determinación de la dinámica demográfica. La experiencia migratoria constituye en Argentina un fundamento social y una fuente inagotable de renovación cultural, que marca la historia de familias y personas y que actualiza permanentemente en la sociedad un debate en torno de los derechos y las garantías que deben reconocerse a la población extranjera. Por su parte, la emigración nativa de los últimos treinta años instala una serie de interrogantes en torno de la magnitud de la pérdida poblacional, de su significación

\footnotetext{
* Magister en Demografía (Universidad Nacional de Luján) y Licenciada en Sociología (Universidad de Buenos Aires), docente investigadora en la Facultad de Ciencias Sociales - Universidad de Buenos Aires (UBA). E-mail: lauracalv@sociales.uba.ar. Buenos Aires/Argentina.
} 
demográfica y social y de los problemas de los argentinos residentes en países centrales donde sus condiciones de vida se ven amenazadas en contextos crecientemente restrictivos de la migración internacional.

El panorama de las fuentes de información para el estudio de la migración internacional en Argentina es muy limitado (Cuadro 1), motivo por el cual cada vez que se difunden los datos de un censo de población se inicia el análisis de la migración que tuvo lugar durante los diez años anteriores a su levantamiento. ${ }^{1}$ Esa cuestión, la del análisis de la migración a partir de información censal referida a stocks sobrevivientes al momento del censo, requiere que se sigan indicando sus potencialidades y limitaciones, asunto comúnmente omitido cuando se toma como actual información de stocks para hacer referencia a los flujos migratorios que tuvieron lugar durante el período intercensal. Por otra parte sigue siendo necesario insistir en que la medición de la migración internacional a partir de un censo de población arroja estimaciones afectadas por determinados tipos de error, ${ }^{2}$ los que deben ser identificados y subsanados - siempre y cuando la corrección se considere necesaria y sea posible. ${ }^{3}$ En todo caso, mientras se trabaje con datos no corregidos de la población inmigrante, debe tenerse eso presente para valorizar los resultados obtenidos como razonables aproximaciones a las estimaciones de la migración internacional antes que como estimaciones definitivas de su cuantum y atributos.

En el mes de octubre de 2010 se levantó en todo el territorio de la República Argentina el décimo Censo Nacional de Población, Hogares y Viviendas 2010. Se trató de un censo de población realizado conforme la metodología de censos de hecho, a la que pertenece la totalidad de los censos de población argentinos, con inclusión de muestreo para la aplicación del cuestionario ampliado en localidades de más de 50.000 habitantes de acuerdo a las proyecciones de población. ${ }^{4}$ La publicación de resultados del censo fue iniciada al año siguiente por el INDEC y sus datos revelan las primeras características de la migración internacional al cabo de una década de desenvolvimiento económico y social, marcado por grandes cambios desde que se levantara el censo de población anterior en noviembre de 2001.

1 CALVELO, Laura. "Inmigración y emigración internacional en Argentina. Problemas de medición y estimación".

2 El error más mencionado es el de omisión, pero existen otros defectos de la información censal que afectan la precisión y las posibilidades de obtener estimaciones insesgadas de los atributos de la migración internacional.

3 La corrección de los defectos de la información proveniente de fuentes secundarias, como los censos de población, requiere disponer de una serie de insumos del análisis demográfico que no siempre se hallan disponibles.

4 INDEC. Censo Nacional de Población, Hogares y Viviendas 2010. 
En el siguiente artículo se presenta una descripción general de la migración internacional en Argentina hacia 2010 desde la perspectiva de los stocks de población a partir de datos censales. En el caso de la población no nativa se basa en información del Censo Nacional de Población, Hogares y Viviendas 2010 tal como fuese publicada por el Instituto de Estadística (INDEC). ${ }^{5}$ En el caso de la migración internacional de la población nativa de Argentina, dada en su caso la prevalencia de la emigración neta, el análisis se basa en información de censos extranjeros hasta la ronda 2000, corregidos y proyectados hasta 2010 de acuerdo a la experiencia acumulada acerca de la evolución de los stocks de argentinos en el exterior y de las fuentes de error que afectan sus estimaciones. ${ }^{6}$ Asimismo se presentan evidencias provenientes de otras fuentes de datos de los principales países de destino (Estados Unidos y España).

La información que se presenta refleja los cambios que tuvieron lugar en la migración internacional a lo largo de la década anterior al levantamiento del Censo de 2010. La década 2000-2010 en Argentina representa el pasaje de un período de inusitada crisis económica y social (hasta 2003 inclusive) a un período de intenso crecimiento económico, recuperación del mercado de trabajo y fortalecimiento de la capacidad estatal (desde 2004 hasta 2010). La nación vivió en esa década uno de los mayores flagelos de su historia, con altísimos niveles de pobreza, indigencia y desocupación, y una de las recuperaciones económicas más aceleradas del último siglo.

El comportamiento de la migración internacional de ese período (hasta 2001) fue de una intensa afluencia de población procedente en su mayor parte de países limítrofes y Perú y de una no menos acelerada emigración de población mayoritariamente nativa. La inmigración procedente de la región se vio fuertemente incentivada por la dolarización de la economía argentina de los años noventa, y se destacó el intenso crecimiento de la inmigración peruana y una característica aparición de cuotas de entradas internacionales

5 Debe tenerse presente que se trata de datos sujetos a errores muestrales que en el caso de la migración internacional se vuelven significativos en la medida en que los universos de análisis sean reducidos, puesto que el país de nacimiento de la población se relevó tanto en el cuestionario básico como en el cuestionario ampliado. Las estimaciones del cuantum de la población inmigrante ofrecidas en este artículo presentan coeficientes de variación inferiores a 10 por ciento, por lo que se consideran suficientemente precisas del fenómeno a tratar. Debe advertirse al mismo tiempo que las variables analizadas del Censo 2010 son completamente íntegras, es decir que tanto la no respuesta como la respuesta incoherente o inespecífica han sido imputadas durante el procesamiento de datos. Hasta el momento se desconocen los criterios de tal asignación, cuya consideración sería valiosa al momento de analizar los resultados.

6 Los volúmenes observados de población nacida en Argentina residiendo en otros países en el año 2010 podrán conocerse cuando se encuentren disponibles los resultados de los censos de población correspondientes a la ronda 2010, la que actualmente se encuentra transitando su etapa de levantamiento. 
de población de países geográficamente lejanos (como algunos del bloque ex-soviético y otros asiáticos). ${ }^{7}$ Pero por otro lado en ese período tuvo lugar una voluminosa emigración nativa en dirección hacia países centrales, principalmente Estados Unidos, Canadá y varios de la Unión Europea (España en primer lugar, pero también Italia, Gran Bretaña y Francia). Ese fenómeno, persistente desde la década anterior, se extendió más allá de 2001, posiblemente incluso hasta 2004-2005, y representa la agudización de la emigración nativa más intensa de la historia del país. ${ }^{8}$

\section{CUADRO 1 \\ Argentina: Panorama de las Estimaciones de la Migración Internacional hacia 2010}

\begin{tabular}{|c|c|c|c|c|c|c|c|c|}
\hline \multirow[b]{2}{*}{ 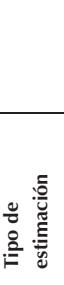 } & \multirow[b]{2}{*}{$\begin{array}{l}\text { Principales } \\
\text { universos }\end{array}$} & \multicolumn{3}{|c|}{ Principales fuentes nacionales } & \multicolumn{2}{|c|}{$\begin{array}{c}\text { Sistemas de } \\
\text { información } \\
\text { migratoria en la } \\
\text { región }\end{array}$} & \multicolumn{2}{|c|}{$\begin{array}{c}\text { Otras fuentes } \\
\text { destacadas a nivel } \\
\text { extrarregional }\end{array}$} \\
\hline & & $\begin{array}{c}\text { Censo } \\
\text { Nacional } \\
\text { de } \\
\text { Población } \\
2010\end{array}$ & $\begin{array}{c}\text { Encuesta } \\
\text { Permanen } \\
\text { te de } \\
\text { Hogares }\end{array}$ & $\begin{array}{l}\text { Registros } \\
\text { de entra- } \\
\text { das y } \\
\text { salidas }\end{array}$ & $\begin{array}{l}\text { IMILA } \\
\text { (censos } \\
\text { de } \\
\text { población } \\
\text { ronda } \\
2010 \text { ) }\end{array}$ & $\begin{array}{l}\text { SICREMI- } \\
\text { ALC } \\
\text { (censos, } \\
\text { encuestas } \\
y \\
\text { registros) }\end{array}$ & $\begin{array}{l}\text { España - } \\
\text { Censo de } \\
\text { población y } \\
\text { Padrón } \\
\text { municipal }\end{array}$ & $\begin{array}{c}\text { Estados } \\
\text { Unidos, } \\
\text { censo de } \\
\text { población } \\
\text { y ACS }\end{array}$ \\
\hline \multirow{3}{*}{ 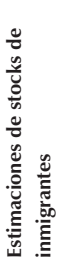 } & $\begin{array}{l}\text { Población no nativa } \\
\text { (migración absoluta) }\end{array}$ & $x$ & $\mathrm{x}$ & - & $x$ & $x$ & - & - \\
\hline & $\begin{array}{l}\text { Población no nativa } \\
\text { que llegó en los } \\
\text { últimos cinco años } \\
\text { (migración reciente) }\end{array}$ & $x$ & $\mathrm{x}$ & - & $\mathrm{x}$ & $\mathrm{x}$ & - & - \\
\hline & $\begin{array}{l}\text { Población nativa de } \\
\text { retorno (migración } \\
\text { reciente) }\end{array}$ & $\mathrm{x}$ & $\mathrm{x}$ & - & $\mathrm{x}$ & $\mathrm{x}$ & - & - \\
\hline \multirow[b]{2}{*}{ 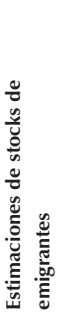 } & $\begin{array}{l}\text { Población nativa } \\
\text { residiendo en el } \\
\text { exterior (migración } \\
\text { absoluta) }\end{array}$ & - & - & - & $\mathrm{x}$ & $\mathrm{x}$ & $\mathrm{x}$ & $x$ \\
\hline & $\begin{array}{l}\text { Población nativa } \\
\text { residiendo en el } \\
\text { exterior, llegada al } \\
\text { país de destino en } \\
\text { los últimos cinco } \\
\text { años (migración } \\
\text { reciente) }\end{array}$ & - & - & - & $\mathrm{x}$ & $\mathrm{x}$ & $x$ & $x$ \\
\hline \multirow{3}{*}{ 蒿 } & Población total & Saldos & - & Balances & - & - & - & - \\
\hline & Población no nativa & migratorio & - & $\begin{array}{c}\text { de } \\
\text { entradas } y\end{array}$ & - & $\mathrm{x}$ & - & - \\
\hline & Población nativa & $2010\left(X^{1}\right)$ & - & salidas $\left(X^{2}\right)$ & - & $x$ & - & - \\
\hline
\end{tabular}

Fuente: CALVELO, Laura. "Inmigración y emigración internacional en Argentina. Problemas de medición y estimación". $\left(\mathrm{X}^{1}\right)$ Estimación de saldos migratorios por métodos indirectos; $\left(\mathrm{X}^{2}\right)$ Estimación de balances de movimientos internacionales de entradas y salidas.

CALVELO, Laura. "Migraciones internas e internacionales en los tiempos del ajuste".

8 IDEM. Crisis y emigración. La emigración de los argentinos entre 1960 y 2002; IDEM. "Estimaciones migratorias en Argentina: La migración neta de nativos posterior a 1980". 
Desde 2004 en adelante la economía argentina creció a un ritmo acelerado $^{9}$ y tuvo lugar un proceso de legitimación del sistema político y de reconstrucción social no exento, por supuesto, de nuevas confrontaciones. El escenario emergente desde entonces parece indicar la continuidad de la inmigración internacional dominada por los países sudamericanos, y cuotas de retorno de la población nativa, lo que indudablemente refleja el impacto de la crítica coyuntura económica en Estados Unidos y los países europeos crecientemente afectados por las consecuencias de la crisis de 2008.

\section{La inmigración no nativa}

La disponibilidad de información del Censo de Población 2010 de Argentina revela que el stock de población extranjera residente del país se incrementó entre 2001 y 2010 un 18 por ciento, pasando de 1,5 a 1,8 millones de personas (Cuadro 2).

\section{CUADRO 2}

Argentina. Población no nativa por país de nacimiento, 2001 y 2010

\begin{tabular}{|c|c|c|c|c|c|}
\hline País de nacimiento & 2001 & & 2010 & & \begin{tabular}{|c|} 
Incremento \\
$2001 / 2010$ \\
(diferencia \%)
\end{tabular} \\
\hline & & $(\%)$ & & $(\%)$ & \\
\hline Total & 1.531 .940 & 100,0 & 1.805 .957 & 100,0 & 17,9 \\
\hline AMÉRICA & 1.041.117 & 68,0 & 1.471 .399 & 81,5 & 41,3 \\
\hline Paises limitrofes & 923.215 & 60,3 & 1.245 .054 & 68,9 & 34,9 \\
\hline Bolivia & 233.464 & 15,2 & 345.272 & 19,1 & 47,9 \\
\hline Brasil & 34.712 & 2,3 & 41.330 & 2,3 & 19,1 \\
\hline Chile & 212.429 & 13,9 & 191.147 & 10,6 & $-10,0$ \\
\hline Paraguay & 325.046 & 21,2 & 550.713 & 30,5 & 69,4 \\
\hline Uruguay & 117.564 & 7,7 & 116.592 & 6,5 & $-0,8$ \\
\hline Países no limitrofes (América) & 117.902 & 7,7 & 226.345 & 12,5 & 92,0 \\
\hline Perú & 88.260 & 5,8 & 157.514 & 8,7 & 78,5 \\
\hline Resto de América & 29.642 & 1,9 & 68.831 & 3,8 & 132,2 \\
\hline EUROPA & 432.349 & 28,2 & 299.394 & 16,6 & $-30,8$ \\
\hline Alemania & 10.362 & 0,7 & 8.416 & 0,5 & $-18,8$ \\
\hline España & 134.417 & 8,8 & 94.030 & 5,2 & $-30,0$ \\
\hline Francia & 6.578 & 0,4 & 6.995 & 0,4 & 6,3 \\
\hline Italia & 216.718 & 14,1 & 147.499 & 8,2 & $-31,9$ \\
\hline Resto de Europa & 64.274 & 4,2 & 42.454 & 2,4 & $-33,9$ \\
\hline ASIA & 29.672 & 1,9 & 31.001 & 1,7 & 4,5 \\
\hline China & 4.184 & 0,3 & 8.929 & 0,5 & 113,4 \\
\hline Corea & 8.205 & 0,5 & 7.321 & 0,4 & $-10,8$ \\
\hline Japón & 4.753 & 0,3 & 4.036 & 0,2 & $-15,1$ \\
\hline Libano & 1.619 & 0,1 & 933 & 0,1 & $-42,4$ \\
\hline Siria & 2.350 & 0,2 & 1.337 & 0,1 & $-43,1$ \\
\hline Taiw án & 3.511 & 0,2 & 2.875 & 0,2 & $-18,1$ \\
\hline Resto de Asia & 5.050 & 0,3 & 5.570 & 0,3 & 10,3 \\
\hline ÁFRICA & 1.883 & 0,1 & 2.738 & 0,2 & 45,4 \\
\hline OCEANÍA & 747 & 0,0 & 1.425 & 0,1 & 90,8 \\
\hline Ignorado & 26.172 & 1,7 & * & * & - \\
\hline
\end{tabular}

9 CEPAL. Estudio económico de América Latina y el Caribe 2010-2011. 
En 2001 la población extranjera constituía el 4,2 por ciento de la población total, valor que hacia 2010 prácticamente no registró cambios significativos en volumen, ubicándose en 4,5 por ciento. Paralelamente se intensificó un fenómeno que trae varias décadas de desarrollo, el incremento del peso de la población nacida en países limítrofes en el conjunto de la población extranjera, Ilegando a constituir en 2010 casi el 70 por ciento de ella (Gráfico 1).

\section{GRÁFICO 1 \\ Argentina. Distribución de la población no nativa según condición limítrofe del país de nacimiento, 1869-2010}

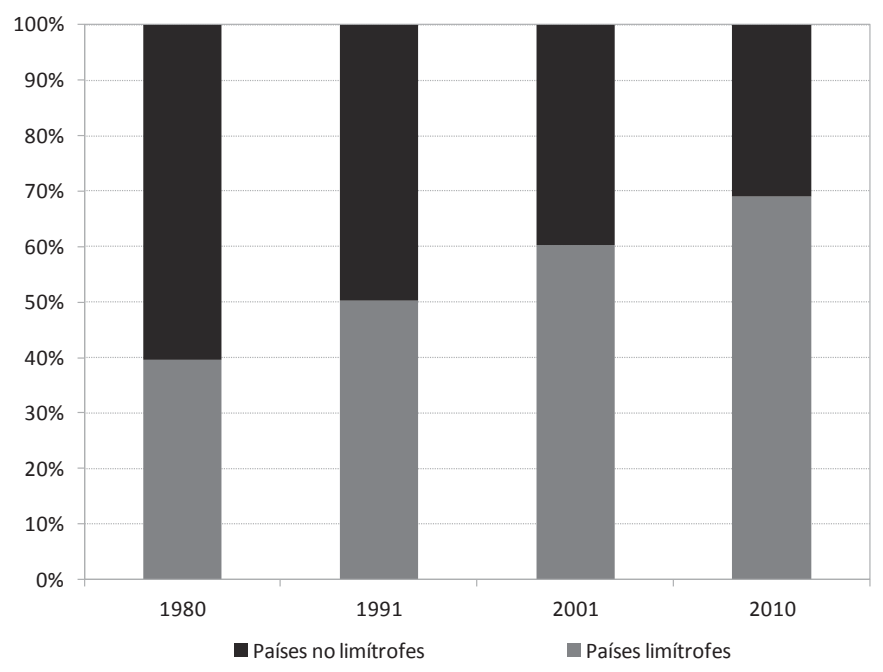

Fuente: INDEC. Censos de población 1980, 1991, 2001 y 2010.

En términos absolutos los incrementos más significativos se registraron entre la población nacida en Paraguay, Bolivia y Perú, principales colectividades de inmigrantes del vigoroso patrón de la migración intrarregional ${ }^{10}$ en Argentina, que registra constantes flujos de inmigrantes jóvenes que lo mantienen en permanente vitalidad; en tanto que las disminuciones más significativas tuvieron lugar entre la población nacida en Italia y España, formada en su mayor parte por los últimos sobrevivientes de antiguas cohortes migratorias correspondientes al patrón de la inmigración ultramarina, que lleva ya más de medio siglo sin renovarse (Gráfico 2).

\footnotetext{
${ }^{10}$ Para el conocimiento de los patrones migratorios prevalecientes en América Latina consúltese MARTÍNEZ PIZARRO, José. "América Latina y el Caribe: migración internacional, derechos humanos y desarrollo".
} 


\section{GRÁFICO 2 \\ Argentina. Población no nativa por país de nacimiento, 2001 y 2010 (en miles)}

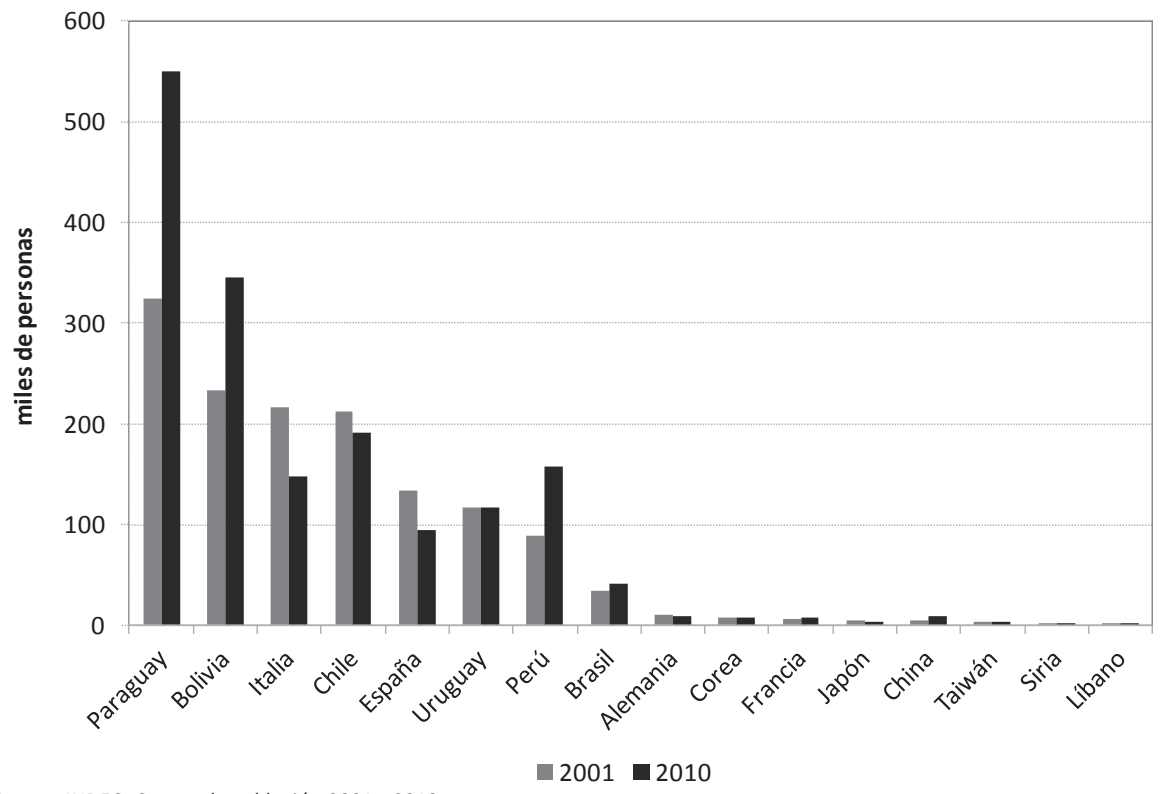

Fuente: INDEC. Censos de población 2001 y 2010

Sin embargo aparecen otras colectividades de inmigrantes menos voluminosas pero que vienen registrando singular dinamismo. En el Gráfico 3 las diferencias porcentuales revelan que la población nacida en China se duplicó entre 2001 y 2010, pasando de 4 mil a casi 9 mil personas. Vale destacar el aumento de la población nacida en Francia, lo que refleja la llegada de población joven, asunto sobre el que existían indicios previos y que se verá más adelante. Como se mencionara más arriba, se destaca la intensidad del incremento de la población nacida en Perú, fenómeno que se venía observando desde la década anterior. ${ }^{11}$ En opuesta situación aparece la población nacida en países de Asia, tales como Siria y Líbano, con significativas reducciones; la población de Italia y España, que habiendo sido voluminosa en el pasado se viene reduciendo por efecto casi exclusivo de la mortalidad; los nacidos en Chile y Uruguay también se reducen, reflejando significativas desaceleraciones de los flujos de inmigración hacia Argentina.

${ }^{11}$ Entre otros, BENENCIA, Roberto. "La inmigración limítrofe". 


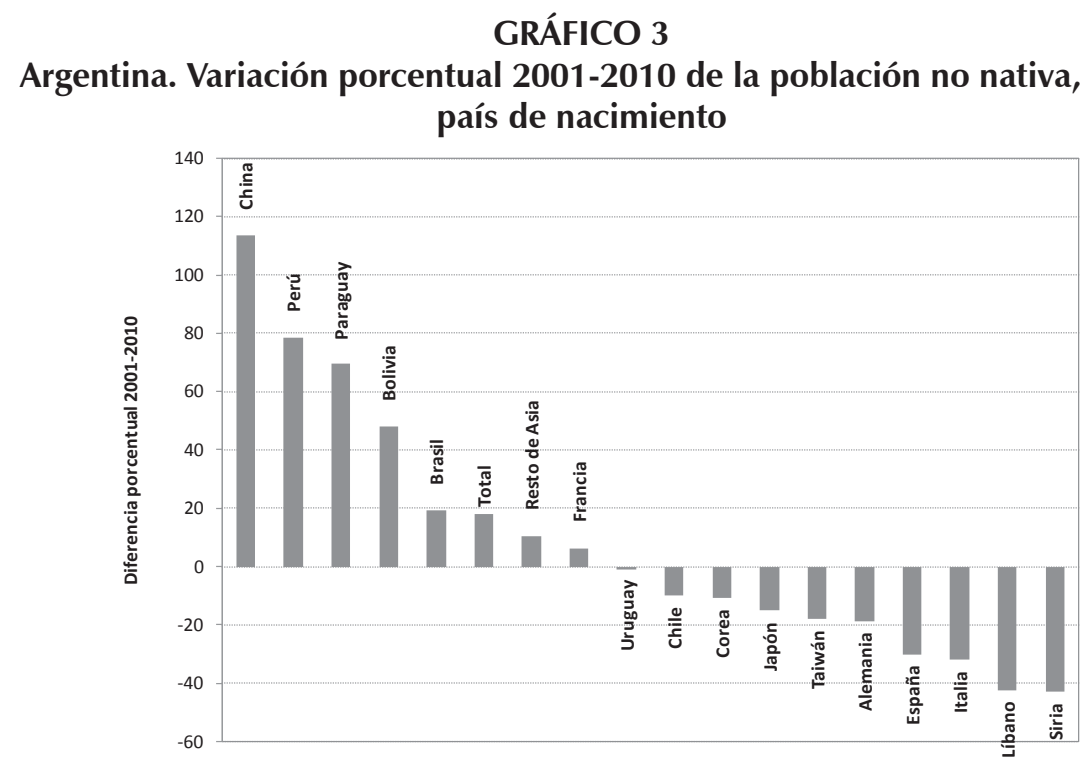

Fuente: INDEC. Censos de población 2001 y 2010

La información del Censo 2010 difundida hasta el momento permite analizar las estructura por sexo y edad de la población no nativa por país de nacimiento a nivel de los grandes grupos etarios (0-14, 15-64 y 65 años y más). Para un análisis de mayor precisión demográfica sólo es posible observar las estructuras por sexo y edad de la población no nativa en relación a la población nativa, lo que se presenta para 2001 y 2010 en los Gráficos 4 a y b.

\section{GRÁFICOS 4 a y b}

Argentina. Pirámides de la población total, nativa y no nativa, 2001 y 2010
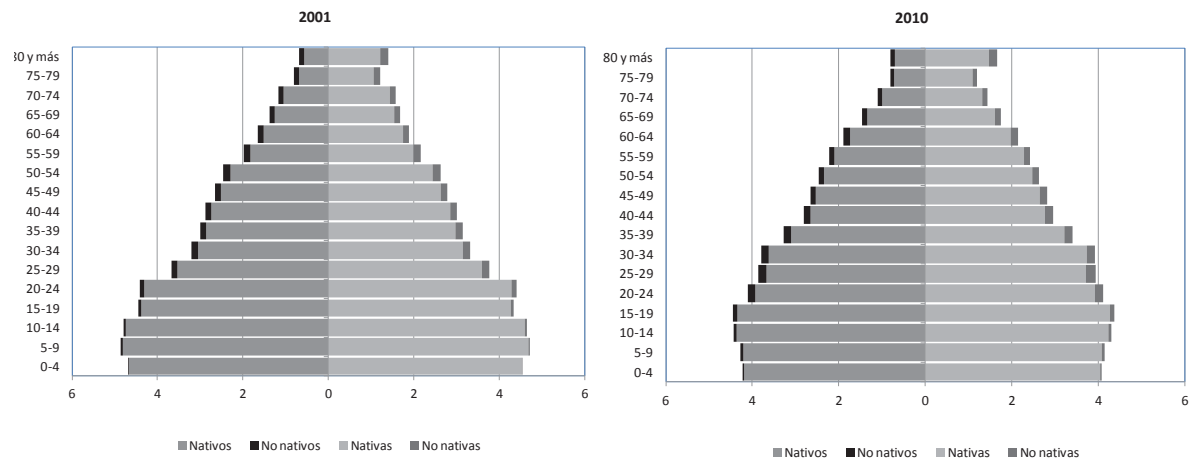

Fuente: INDEC, Censos de población 2001 y 2010. 
El detalle de la estructura por sexo y edad de la población no nativa entre 2001 y 2010 revela mayor información (Gráficos 5 a y b). Tomando en consideración que los datos presentados no incluyen correcciones, se señala la mayor presencia de niños de hasta 9 años de edad, e incluso del grupo de 15 a 19 años. Al mismo tiempo se observan incrementos tanto en varones como en mujeres en las edades centrales de 20 a 29 años. El conjunto de modificaciones señala la persistente renovación de los flujos migratorios provenientes de la región latinoamericana, principalmente población nacida en Paraguay, Bolivia y Perú, pero también señala un cambio técnico derivado del cambio de contexto normativo de la migración internacional en Argentina ${ }^{12}$. Por último, el significativo peso de población en edades muy avanzadas, principalmente mujeres, denota la sobrevivencia de población perteneciente a cohortes migratorias anteriores a mitad del siglo XX, en su mayor parte procedentes de países europeos.

\section{GRÁFICOS 5 a y b \\ Argentina. Pirámides de la población no nativa, 2001 y 2010}
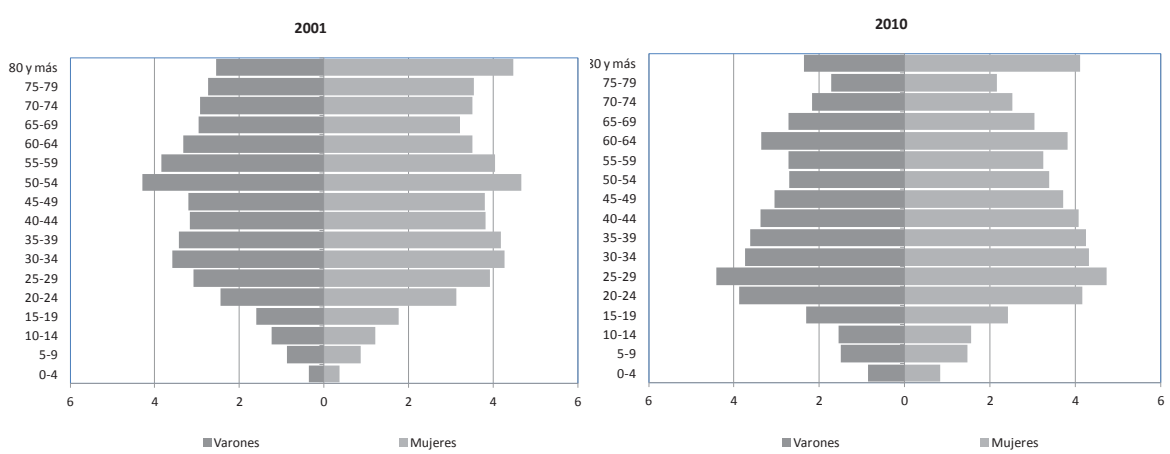

Fuente: INDEC, Censos de población 2001 y 2010.

El avanzado envejecimiento de la población de países de Europa se evidencia en el Gráfico 6, donde también se observa la mayoritaria presencia de población en edades centrales, de intensa participación en el mercado de trabajo, propia de la estructura de la población de países americanos. En tal sentido los Gráficos 7 a, b y c aportan precisiones. En el caso de los países de América, limítrofes y Perú, se confirman las observaciones anteriores, con notorias

12 Desde que la Ley 25.871 fuese sancionada en diciembre de 2003 tuvo lugar una transformación en las condiciones de admisión de la población extranjera, particularmente proveniente de países de Sudamérica, facilitándose su regularización documentaria. El fuerte impacto de la regularización tiene un positivo efecto sobre la disposición de la población extranjera a declarar su país de nacimiento, lo que se traduce en menores cuotas de subenumeración censal. 
presencias de niños en los países americanos y predomino de población adulta mayor en el caso de los europeos. La situación de los países asiáticos es mixta en este sentido, apareciendo casos de agudo envejecimiento de la estructura (Japón) y otros de que indican la presencia de flujos más recientes (China).

\section{GRÁFICO 6 \\ Argentina. Distribución por grupos funcionales de edad de la población no nativa según región de nacimiento, 2010}

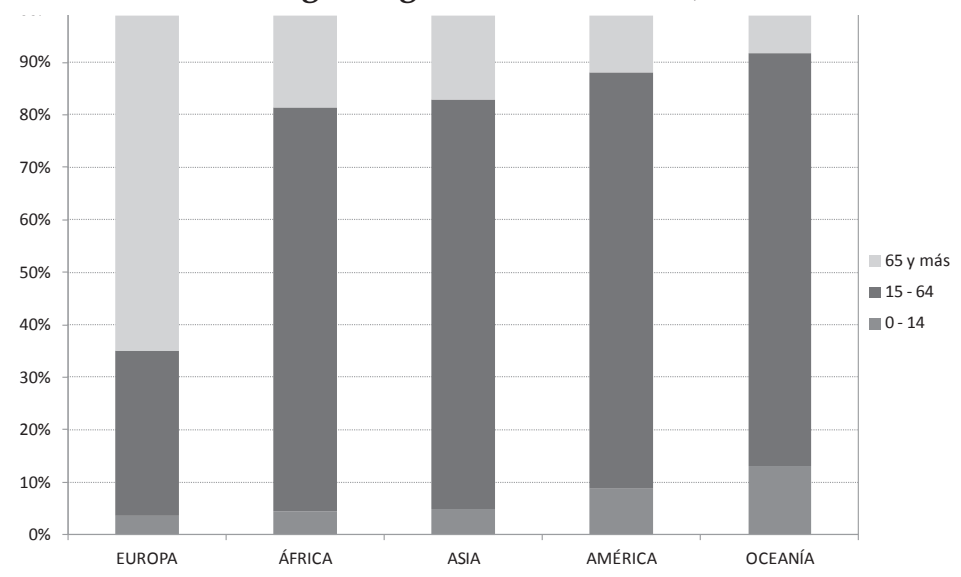

Fuente: INDEC. Censo de población 2010

Particular atención requieren los casos de la población procedente de Francia y Alemania. Si bien presentan estructuras con signos de envejecimiento, también incluyen población infantil y joven llegada en los últimos diez años, lo que indica el efecto renovador de flujos inmigratorios recientes. Ese fenómeno también se observa en la estructura de la población nacida en España. Sobre estas cualidades ténganse presente dos asuntos. Por una parte la vigencia de flujos de carácter global de población joven de países altamente desarrollados que, al menos hasta mediados de la década 2000, se dirigía a países con sensibles carencias económicas y sociales. Por otra parte el efecto de las contracorrientes de la migración internacional: la significativa presencia de argentinos en países de Europa y Estados Unidos implica el desarrollo de flujos migratorios de retorno que incluyen cónyuges e hijos extranjeros. ${ }^{13}$

\footnotetext{
${ }^{13}$ En ese sentido la información del Censo de Población de 2010 difundida hasta el momento no permite avanzar más allá. Deberán esperarse más detalles para confirmar estas hipótesis con mayor precisión.
} 


\section{GRÁFICO 7 a, b y c}

Argentina. Distribución por grupos funcionales de edad de la población no nativa según país de nacimiento, 2010

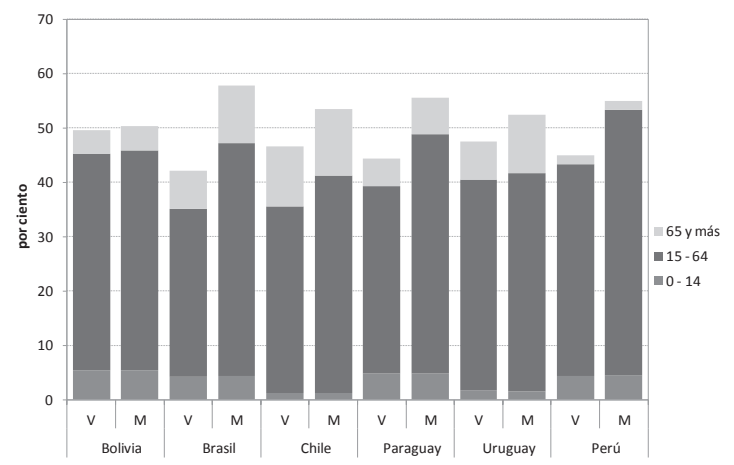

Fuente: INDEC. Censos de población 2010

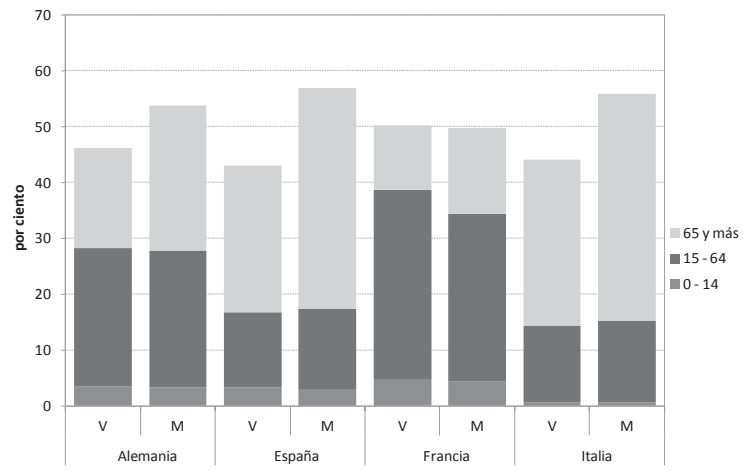

Fuente: INDEC. Censos de población 2010

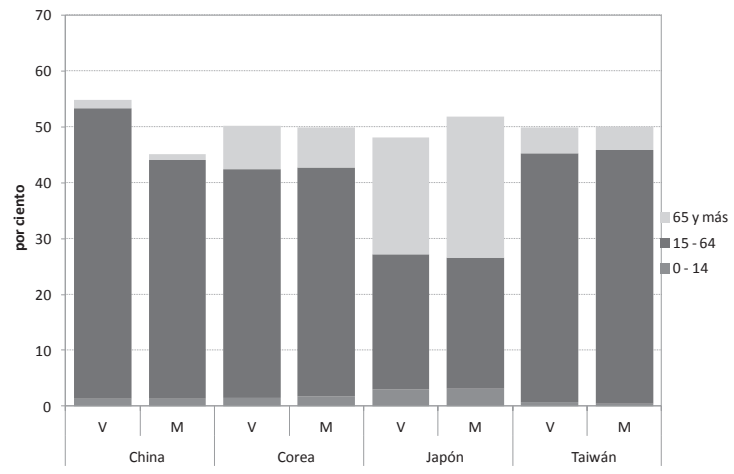

Fuente: INDEC. Censos de población 2010 
La composición por sexo de la población no nativa (Gráfico 8) demuestra un excedente de mujeres en el caso de la población de países europeos y de América por motivos completamente distintos. En el caso de los países de Europa se trata de la sobrevida femenina entre los sobrevivientes de cohortes migratorias antiguas. En el caso de los inmigrantes de países americanos, en su mayor parte limítrofes y Perú, se trata del efecto de la feminización de los flujos migratorios recientes. La feminización de los flujos migratorios ha sido señalada como generalidad de los movimientos de población en nuestro continente. ${ }^{14}$ La elevación de las cuotas de población femenina que migra internacionalmente y del incremento de su modalidad de migración autónoma, son fenómenos vinculados con las repercusiones de los cambios estructurales tanto en las sociedades emisoras como receptoras, asuntos extensos que no desarrollaremos aquí.

\section{GRÁFICO 8 \\ Argentina. Índice de masculinidad de la población no nativa por región de nacimiento, 2001 y 2010}

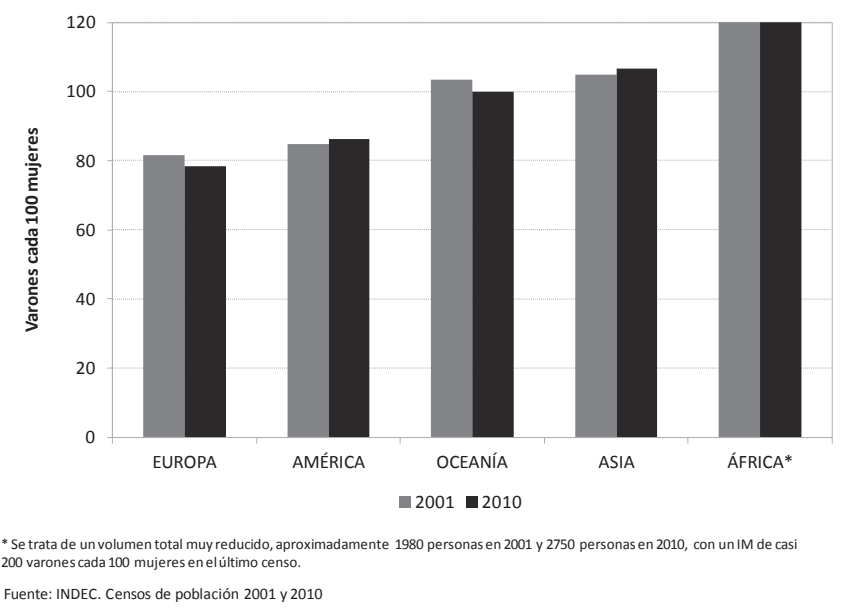

Las mujeres inmigrantes ya habían pasado de constituir el 50 al 54 por ciento entre 1980 y 2001. Esta creciente representación femenina se vinculaba tanto con un aumento general en la proporción de mujeres en las colectividades extranjeras como con el aumento de grupos migratorios en los que tradicionalmente la presencia femenina había sido más elevada, como el

\footnotetext{
${ }^{14}$ PELLEGRINO, Adela. "La migración internacional en América Latina y El Caribe: tendencias y perfiles de los migrantes"; MARTÍNEZ PIZARRO, Jorge. América Latina y el Caribe: migración internacional, derechos humanos y desarrollo.
} 
caso de la migración paraguaya y peruana. Los inmigrantes peruanos tienen la mayor proporción de mujeres. En 2001, casi seis de cada diez residentes peruanos en la Argentina eran mujeres, proporción que fue creciendo a lo largo del tiempo. En situación similar aparecían los paraguayos, con una proporción de mujeres también cercana al 60 por ciento. En el caso de la migración paraguaya la representación femenina en las distintas cohortes migratorias siempre superó a los varones. Entre quienes llegaron en los años ochenta las mujeres representan el 59 por ciento y entre quienes arribaron durante los noventa el 57 por ciento.

El año de llegada de la población no nativa permite distinguir la inmigración reciente respecto de los sobrevivientes de antiguas cohortes migratorias. El censo 2010 no relevó el año de llegada sino períodos de llegada determinados (antes de 1991, entre 1991 y 2001, entre 2002 y 2010), cuya observación resulta de mayor interés para el período 2002-2010. Una importante observación es que esta variable, históricamente una de las más defectuosas de los censos de población por su alto nivel de no respuesta, en el Censo 2010 ha sido imputada en su totalidad, desconociéndose hasta el momento los criterios de asignación de la no respuesta y de las respuestas incoherentes.

La migración más reciente (porcentaje de llegados entre 2002 y 2010) se concentra entre la población de Perú, Paraguay y Bolivia, en todos los casos con proporciones superiores al 30 por ciento (Gráfico 9). Aunque en cuotas de volumen mucho más reducido, en ese grupo aparece la población de China y Francia. Tal como se esperara, Chile y Uruguay denotan la menor proporción de llegadas recientes, lo mismo que Italia y España, sobre los que se ya se ofrecieron precisiones.

Hemos visto que tanto en volumen absoluto como en dinamismo reciente la inmigración limítrofe y peruana dominan el panorama de la migración internacional en Argentina. A pesar de que acompaña la historia demográfica del país desde su formación, esa migración adquirió visibilidad a partir de la década del cincuenta no sólo por su aumento en números absolutos sino también por su creciente concentración en el Área Metropolitana de Buenos Aires - AMBA -, el mayor aglomerado urbano del país.

A partir de los años treinta los inmigrantes de provenientes de los países vecinos proporcionaron una solución a la escasez de mano de obra que las economías fronterizas venían padeciendo en el sector primario de la actividad económica. Las tareas de cosecha y de recolección de cultivos se habían hecho extensivas a la mayor parte del año y junto a la demanda de 
trabajadores no calificados en las zonas urbanas los inmigrantes contaban con trabajo para los doce meses del año. ${ }^{15}$

\section{GRÁFICO 9}

\section{Argentina. Porcentaje de población no nativa llegada a la Argentina entre 2002 y 2010, por país de nacimiento, 2010}

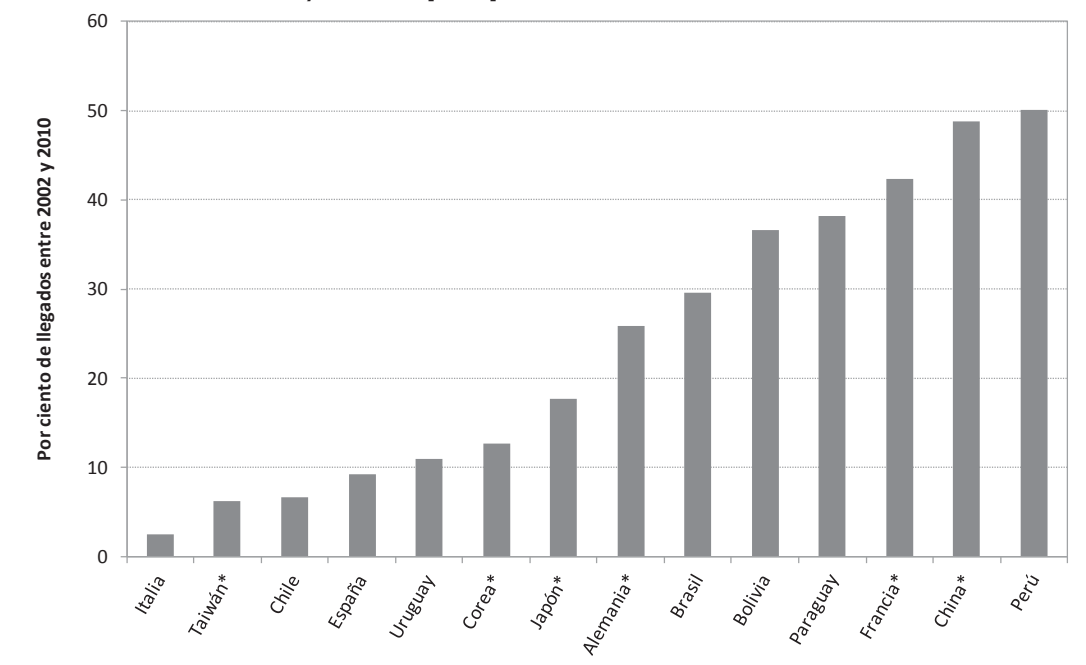

* Universo inferior a 10 mil casos

Fuente: INDEC. Censos de población 2010

Hacia mediados de la década del sesenta, la contratación de mano de obra limítrofe quedó subordinada al comportamiento de la migración interna. La incorporación de tecnología que implicaba el ahorro de mano de obra y la caída de los precios de los productos regionales contribuyeron a que cada vez más inmigrantes limítrofes se trasladaran en busca de oportunidades laborales especialmente en el área de la construcción, concentrada en el Area Metropolitana de Buenos Aires. Así, en una primera larga etapa histórica, la población de origen limítrofe se localizaba en las provincias fronterizas con sus países, en tanto que posteriormente incrementaron su concentración en el AMBA.

Un caso a destacar es el de los inmigrantes peruanos que se han venido incrementando de manera sostenida desde la década del noventa hasta transformarse según los datos del Censo 2001 en la cuarta comunidad de inmigrantes más numerosa de Argentina. La población peruana reside prioritariamente en el AMBA, y posee la singularidad de ser una inmigración

\footnotetext{
${ }^{15}$ BENENCIA, op. cit.
} 
predominantemente femenina y de un nivel educativo alto: según datos de 2001 un 40\% tenía educación secundaria y algo más de la mitad de los integrantes de este grupo tenía estudios técnicos o universitarios. ${ }^{16}$

Históricamente, la proximidad geográfica y las oportunidades laborales en las provincias fronterizas fueron centrales para explicar los patrones de residencia de los inmigrantes de países limítrofes. La migración paraguaya se concentraba a principios del siglo XX principalmente en Misiones, Corrientes y Formosa y sólo una minoría en Buenos Aires. Sin embargo, con el correr del tiempo los inmigrantes paraguayos se fueron concentrando en el AMBA.

Entre los inmigrantes bolivianos se consolida también el patrón de concentración en Buenos Aires. Al igual que los inmigrantes de origen paraguayo, los bolivianos comenzaron diversificando sus destinos y luego se concentraron en el AMBA. Hasta 2001 sólo el 22 por ciento de los inmigrantes bolivianos residían en Salta y Jujuy. Estos cambios en los patrones de asentamiento se vinculan con la crisis de las economías regionales, la reconversión tecnológica de la agricultura y la atracción ejercida por el AMBA.

Los inmigrantes de Chile, quienes llegaron en su mayoría varias décadas atrás, residen mayormente en provincias fronterizas, Chubut, Río Negro, Neuquén y Santa Cruz. En cuanto a los peruanos, que representan una migración más reciente, se han concentrado en la Ciudad de Buenos Aires, y en menor medida en los partidos de la Provincia de Buenos Aires. Finalmente los inmigrantes uruguayos presentan la mayor concentración en Buenos Aires, particularmente en la Provincia de Buenos Aires. Nueve de cada diez inmigrantes uruguayos reside en la Ciudad o en la Provincia de Buenos Aires.

Los Mapas 1 a y $b^{17}$ presentan la distribución de la población no nativa total por provincia en 2010, en tanto que los Mapas 2 a y b ofrecen la distribución por departamento. Interesa señalar la mayor concentración de inmigrantes internacionales en torno del AMBA y de las regiones patagónica (Chubut, Neuquén, Río Negro, Santa Cruz y Tierra del Fuego) y norte del país, tanto noroeste (Salta y Jujuy) como noreste (Formosa y Misiones).

16 Ibidem.

17 El Mapa 1 b es una vista ampliada de la Ciudad Autónoma de Buenos Aires, antiguamente Capital Federal del país, que de otro modo no llega a visualizase por su reducida superficie en el Mapa 1 a (del territorio nacional en conjunto). El Mapa 2 b es una vista ampliada de la Ciudad Autónoma de Buenos Aires y de los Partidos del Gran Buenos Aires, que en conjunto forman el Area Metropolitana de Buenos Aires (AMBA). 
MAPA 1 a y b

Argentina. Porcentaje de población no nativa por provincia, 2010

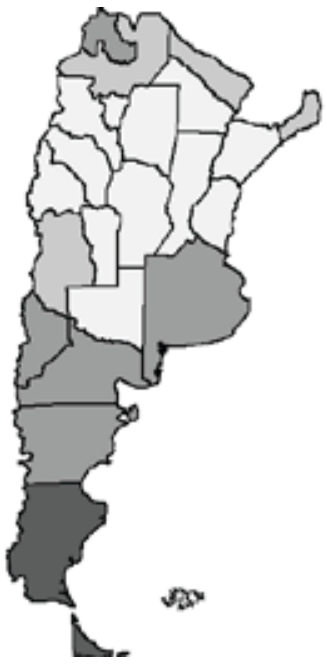

Fuente: INDEC. Censo de población 2010
Vista ampliada de Ciudad Autónoma de Buenos Aires
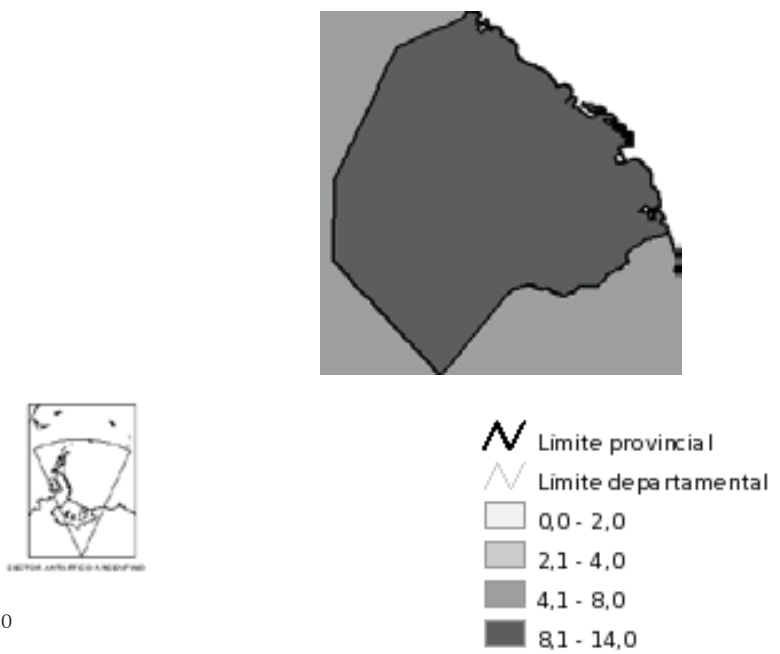

MAPA 2 a y b

Argentina. Porcentaje de población no nativa por departamento, 2010

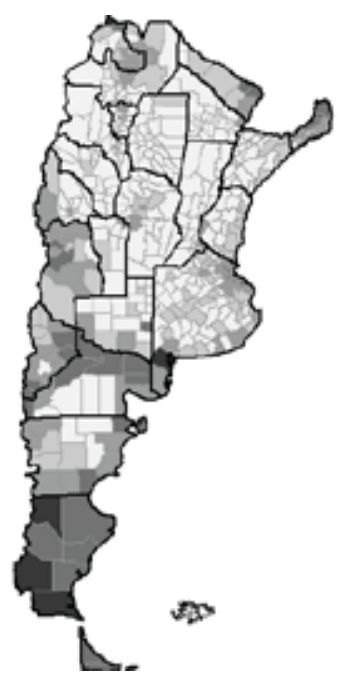

Fuente: INDEC. Censo de población 2010
Vista ampliada de Ciudad Autónoma de Buenos Aires y 24 partidos del Gran Buenos Aires
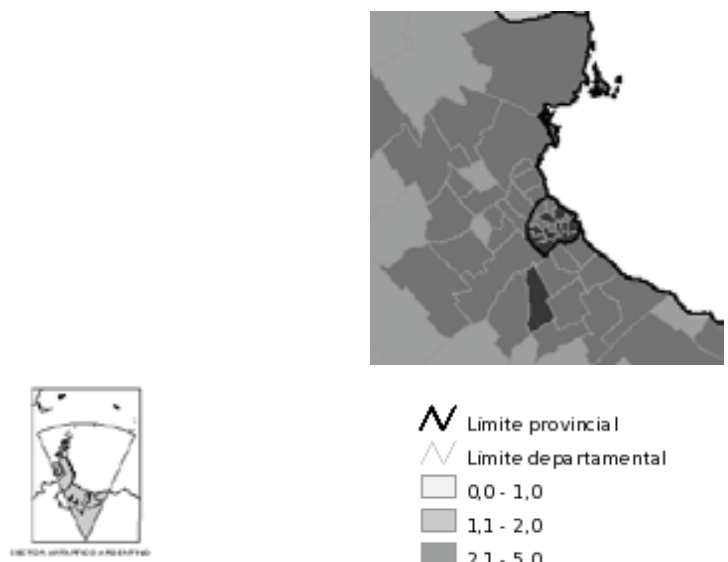

$\wedge$ Limite provincial

Limite depa rtamental

$0,0 \cdot 1,0$

$1,1 \cdot 2,0$

$2,1 \cdot 5,0$

$5,1 \cdot 10,0$

$10,1 \cdot 25,0$ 


\section{La emigración nativa}

A partir del estudio de las series históricas de argentinos en el exterior ${ }^{18}$ y de las fuentes de error que afectan a cada tipo de fuente, pueden elaborarse estimaciones de sus stocks por grandes regiones hacia 2010, que revisten el carácter de aproximaciones a órdenes de magnitud en tanto se espera que se encuentren disponibles sus recuentos en los censos de población de los principales países receptores. ${ }^{19}$ La estimación de argentinos en el exterior al año 2010 que se presenta a continuación se realizó por métodos aritméticos (proyecciones del crecimiento) considerando las condiciones de contexto internacional y local, y partiendo de la evolución observada de los stocks entre 1960 y 2000 según datos censales de los países de destino. Se ha considerado que el proceso emigratorio argentino tuvo continuidad entre 2000 y 2010, combinando fluctuaciones de los flujos salientes (correspondientes en su mayor parte a partidas de nuevos emigrantes) y entrantes (correspondientes a retornos de emigrantes anteriores) y llevando paulatinamente a la declinación de la emigración, la que se agotaría en un momento futuro, cuestión sobre la que no pueden ofrecerse más que razonables conjeturas.

Se ha supuesto que los diferenciales de crecimiento observado por región hasta 2000 se habrían mantenido entre 2000 y 2010, lo que implica confiar en a) la vigencia que en la década habría tenido el patrón de destino extrarregional y b) el dinamismo que dentro del mismo habrían sostenido los países de Europa como destino preponderante de los argentinos en épocas recientes. La estimación de los stocks se realizó a nivel regional (América del Norte, América Latina, Europa y Resto del mundo), suponiendo una hipótesis de progresión de las tasas de crecimiento que tenderían linealmente a cero al cabo de 15 años de desaceleración. De tal forma se considera razonable la cifra de 1,026 millones de argentinos en el exterior al año 2010, según la distribución por grandes regiones que se observa en el Cuadro 3.

Evidencias provenientes de fuentes de datos de Estados Unidos y España aportan sustento a la estimación obtenida. El cotejo de resultados se ha realizado con datos del Padrón Municipal de España y de la American Community Survey -ACS-de Estados Unidos, cuya utilidad ha sido materia de análisis. ${ }^{20}$

\footnotetext{
${ }^{18}$ Cada vez que se indica argentinos en el exterior se hace referencia a población nacida en Argentina que reside habitualmente en países extranjeros.

${ }^{19}$ CALVELO, Laura. "Tendencias y patrones de la emigración argentina entre 1960 y 2010".

20 IDEM, “Inmigración y emigración..., op.cit.
} 


\section{CUADRO 3}

Población nacida en Argentina residente en el exterior, por región de residencia, estimada a 2010

\begin{tabular}{lcc}
\cline { 2 - 3 } & 1.026 .000 & $100 \%$ \\
\hline América Latina & 254.000 & $24,7 \%$ \\
América do Norte & 220.000 & $21,4 \%$ \\
Europa & 374.000 & $36,5 \%$ \\
Resto do Mundo & 178.000 & $17,4 \%$ \\
\hline
\end{tabular}

Fuente: CALVELO, Laura. "Inmigración y emigración internacional en Argentina. Problemas de medición y estimación".

Las cifras padronales españolas señalan una presencia de nacidos en Argentina en 2010 del orden de los 300 mil (Gráfico 12), en tanto que en Estados Unidos rondarían los 170 mil (Gráfico 10), valores coherentes con los rangos de magnitud de la estimación. Respecto de las cifras de las fuentes mencionadas no deben perderse de vista algunas observaciones. Las cifras padronales pueden estar sobrerrepresentando la presencia de argentinos, en tanto que no existen motivos para suponer lo mismo de la ACS. Desde que la recesión en la UE diera inicio a una nueva salida de inmigrantes de España, aparecen algunas incertezas sobre la actualización de los registros del padrón para dar seguimiento a los stocks de población extranjera y no extranjera nacida en países de América Latina. ${ }^{21}$

Entre los defectos conocidos de los datos provenientes de esa fuente (tanto sobrerregistro como subregistro) podría incrementar su peso el sobrerregistro, en la medida en que no se reflejen suficientemente las bajas correspondientes a inmigrantes que dejan España. La ACS, en cambio, una fuente de carácter estrictamente estadístico, mantiene la representatividad de sus muestras para el estudio de la inmigración internacional, e incluso aumentó la precisión de sus estimaciones para las desagregaciones por país de nacimiento.

El Gráfico 11 presenta la distribución estimada de los argentinos por estado, demostrando que casi el 60 por ciento de ellos se encuentra en orden de importancia en Florida, California y New York.

\footnotetext{
${ }^{21}$ Recuérdese la importancia cuantitativa de los argentinos residentes en España con nacionalidad española. En la normativa española dicha población no forma parte de la población extranjera.
} 


\section{GRÁFICO 10}

Estados Unidos. Población nacida en Argentina residente en Estados Unidos. Estimaciones 2002-2009 (ACS)

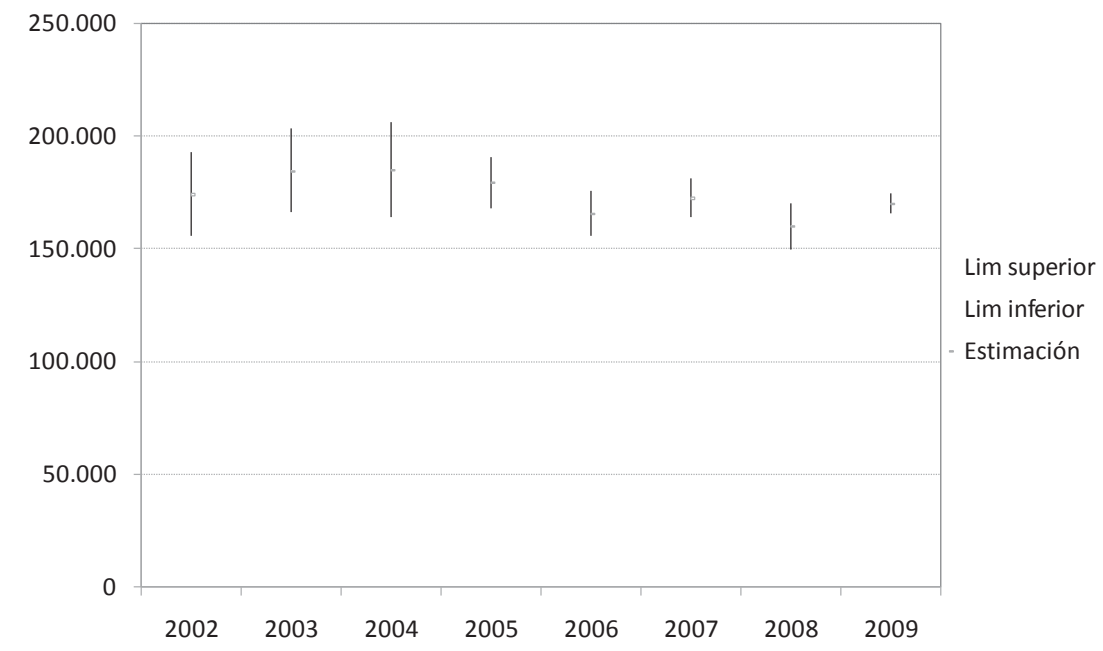

Fuente: Estimaciones propias en base a U.S. Census Bureau 2009 American Community Survey (ACS)

\section{GRÁFICO 11}

Estados Unidos. Población nacida en Argentina, por estado. Estimaciones 2005-2009 (ACS)

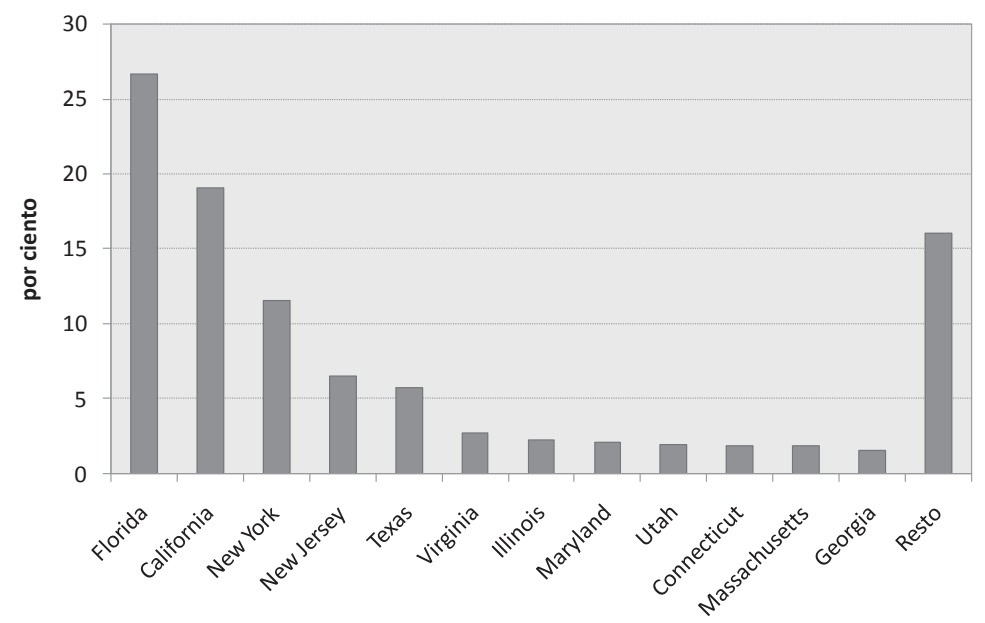

Fuente: Estimaciones propias en base a U.S. Census Bureau 2009 American Community Survey (ACS) 


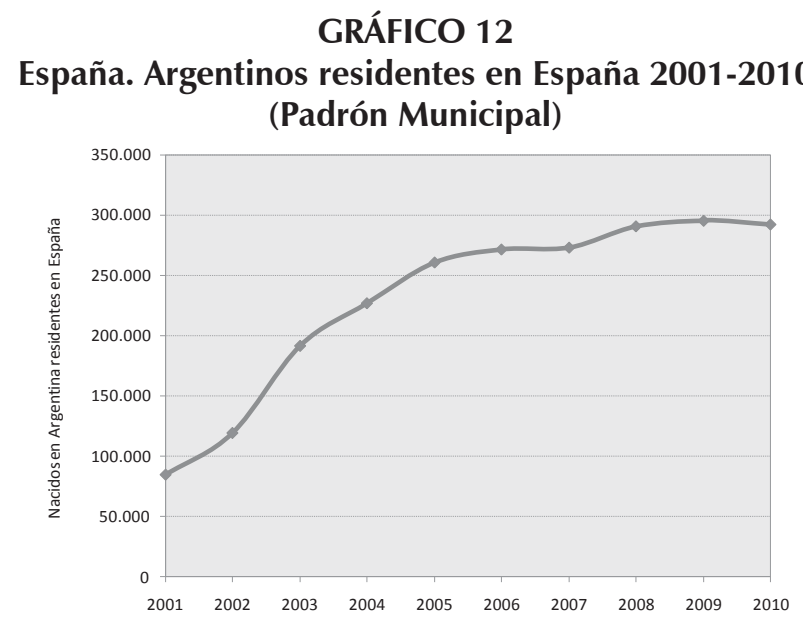

Fuente: Padrón Municipal. INE España. Serie referida al 1 de enero de cada año.

La estimación de argentinos en el exterior presentada para el año 2010 implicaría que su porcentaje respecto de la población total de Argentina se habría colocado entre el 2 y el 3 por ciento, valor calculado respecto de la población total proveniente de los resultados del Censo Nacional de Población, Hogares y Viviendas 2010. Dicha cifra debe considerarse como primera aproximación a la determinación del porcentaje de argentinos en el exterior en la población de Argentina. La incorporación del porcentaje estimado de argentinos en el exterior en 2010 a la serie histórica preexistente, ofrece el siguiente panorama (Gráfico 13) e ilustra el incremento sostenido de sus stocks.

\section{GRÁFICO 13}

Porcentaje de argentinos en el exterior respecto de la población total de Argentina (1960-2010)

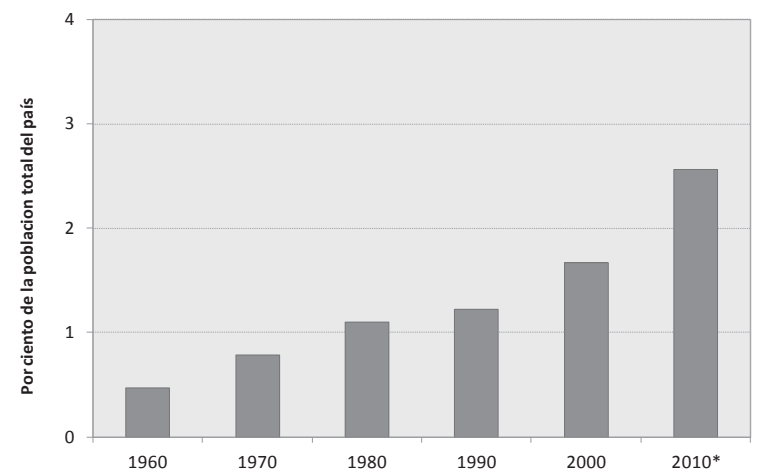

Fuentes: años 1960 a 2000: Calvelo, Laura. CALVELO, Laura. "La emigración en Argentina: 1960-2000". Año 2010: CALVELO, "Inmigración..., op. cit. - * estimación. 
El escenario de las estimaciones al año 2010 prevé el incremento del peso cuantitativo de los emigrantes argentinos en el conjunto de los emigrantes de América Latina, llevando a Argentina a ocupar un lugar entre países que ya en 2000 rondaban el millón de emigrantes, entre los que se encontraban Colombia, Cuba, El Salvador y República Dominicana. En tanto que en términos relativos Argentina se aproximaría a países como Costa Rica, Perú, Chile y Colombia, que en el año 2000 tenían entre un 2 y un 3 por ciento de sus nativos en el exterior.

Por último, de mantenerse los supuestos asumidos en la estimación realizada, podría esperarse el siguiente panorama de distribución de los argentinos en el exterior por grandes regiones desde 1960 y hasta alrededor de 2010 (Gráfico 14).

\section{GRÁFICO 14}

Población argentina residente en el exterior por grandes regiones. Revisiones y estimaciones 1960-2010.

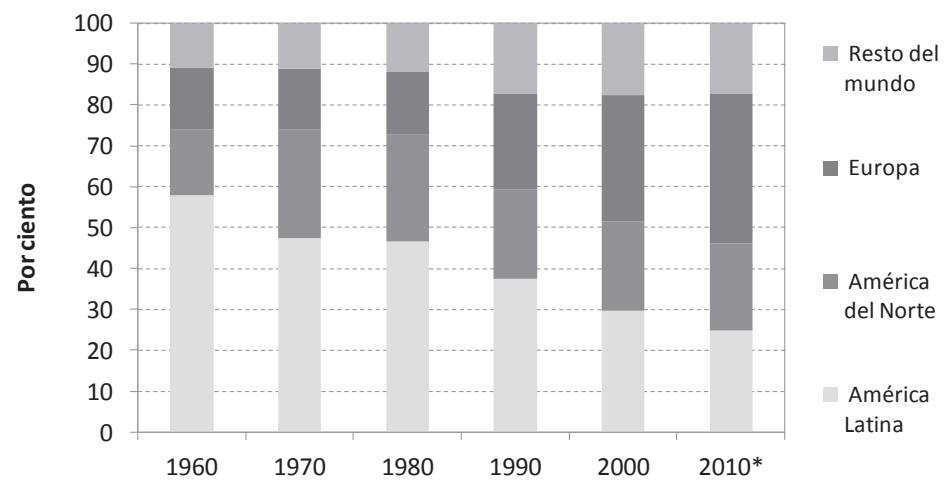

Fuentes: Años 1960 a 2000: CALVELO, Laura. "La emigración en Argentina: 1960-2000"; Censos de población. Año 2010: CALVELO, “Inmigración..., op. cit. - * estimación.

Hasta la portentosa crisis de gobernabilidad de diciembre de 2001, Argentina parecía ser solamente un país de inmigración internacional. En aquellos días de desconcierto y frustración colectiva tuvo lugar el reconocimiento de la condición de Argentina como país de emigración. Desde entonces ha seguido siendo un país de inmigración y de emigración, en el contexto de acelerados cambios de condiciones tanto internacionales como internas. El empeoramiento del contexto mundial y el cambio de signo de la situación nacional abren interrogantes sobre el curso futuro de los movimientos internacionales de población en el país. 


\section{Bibliografia}

BENENCIA, Roberto. "La inmigración limítrofe", in DEVOTO, Fernando. Historia de la inmigración en la Argentina. Buenos Aires: Editorial Sudamericana, 2003.

CACOPARDO, María Cristina; MAGUID Alicia; MARTíNEZ Rosana. "La nueva emigración de latinoamericanos a España: el caso de los argentinos desde una perspectiva comparada", in II Congreso de la Asociación Latinoamericana de Población, 3 al 5 de septiembre de 2006. Guadalajara, 2006.

CALVELO, Laura. Crisis y emigración. La emigración de los argentinos entre 1960 y 2002. Buenos Aires: Ministerio del Interior, 2011.

. "Estimaciones migratorias en Argentina: La migración neta de nativos posterior a 1980", in Revista Notas de Población, n. 91, Santiago de Chile, 2010.

. "Inmigración y emigración internacional en Argentina. Problemas de medición y estimación", in XI Jornadas Argentinas de Estudios de Población (AEPA). 21 al 23 de septiembre de 2011. Ciudad de Neuquén, 2011.

. "La emigración en Argentina: 1960-2000", in TORRADO, Susana (comp.). Población y bienestar en la Argentina del primero al segundo Centenario. Tomo I. Buenos Aires: Edhasa, 2007.

. "Migraciones internas e internacionales en los tiempos del ajuste", in TORRADO, Susana (comp.). El costo social del ajuste. Tomo I. Buenos Aires: Edhasa, 2010.

. "Tendencias y patrones de la emigración argentina entre 1960 y 2010", in LUCHILO, Lucas (coord.). Más allá de la fuga de cerebros. Movilidad, migración y diásporas de argentinos calificados. Buenos Aires: EUDEBA, 2011.

CEPAL. Estudio económico de América Latina y el Caribe 2010-2011. División de Desarrollo Económico. Santiago de Chile: CEPAL, 2011.

CORTÉS, Rosalía; GROISMAN, Fernando. "Migraciones, mercado de trabajo y pobreza en el Gran Buenos Aires", in Revista de la CEPAL, n. 82. CEPAL. Santiago de Chile, 2004.

INDEC. Censo Nacional de Población, Hogares y Viviendas 2010. Anexo metodológico. Resultados definitivos. Variables seleccionadas de los cuestionarios básico y ampliado. Buenos Aires: INDEC, 2012.

MAGUID, Alicia. "La migración internacional en el escenario del MERCOSUR: cambios recientes, asimetrías socioeconómicas y políticas migratorias", in Estudios Migratorios Latinoamericanos, n. 57, Diciembre 2005, 2005.

MARTÍNEZ PIZARRO, Jorge. "Medición e información sobre la migración internacional a partir de los censos: lecciones, desafíos y oportunidades", in Notas de Población, n. 88, año XXXVI, 2009.

- América Latina y el Caribe: migración internacional, derechos humanos y desarrollo. Capítulo II. "Características de la migración internacional en América 
Latina y el Caribe". Santiago de Chile: Comisión Económica para América Latina y el Caribe, 2008.

PELLEGRINO, Adela. "La migración internacional en América Latina y El Caribe: tendencias y perfiles de los migrantes", in CELADE, Serie Población y Desarrollo, n. 35. Santiago, Chile, 2003.

\section{Abstract}

\section{International Migration in Argentina by 2010}

This paper presents the main lines of international migration in Argentina by 2010, focusing in the period between 2000 and 2010. Regarding nonnative immigration, it is based on information from national population censuses of 2001 and 2010. In the case of international migration of Argentinian native population, the analysis is based on foreign censuses until 2000 and other data sources of the main destination countries until 2010 (USA and Spain).

Keywords: International migration in Argentina; Non-native immigration in Argentina; Native emigration in Argentina; Measuring international migration in Argentina.

Recibido para publicación en 25/08/2012. Aceptado para publicación en 29/10/2012.

Received for publication in August, 25 th 2012. Accepted for publication in October, 29 2012. 